\title{
A New Algorithm for Motion Control of Leader-Follower Formation of Mobile Autonomous Agents
}

\author{
Debabrata Atta \\ Research Scholar (PhD) \\ Electrical Engineering Department \\ National Institute of Technology \\ Rourkela, India
}

\author{
Bidyadhar Subudhi \\ Professor \\ Electrical Engineering Department \\ National Institute of Technology \\ Rourkela, India
}

\begin{abstract}
This paper presents a motion control strategy for a rigid and constraint consistent formation that can be modeled by a directed graph whose each vertex represents individual agent kinematics and each of directed edges represents distance constraints maintained by an agent, called follower, to its neighbouring agents. A rigid and constraint consistent graph is called persistent graph. A persistent graph is minimally persistent if it is persistent and no edge can be removed without losing its persistence. An acyclic (free of cycles in its sensing pattern) minimally persistent graph of Leader-Follower structure has been considered here and which can be constructed from an initial Leader-Follower seed (initial graph with two vertices, one is Leader and another one is First Follower and one edge in between them is directed towards Leader) by Henneberg sequence (a procedure of growing a graph) containing only vertex additions. A set of nonlinear optimization based decentralized control laws for mobile autonomous point agents in plane have been proposed. An infinitesimal deviation in formation shape created by continuous motion of Leader is compensated by corresponding continuous motion of other agents fulfilling the shortest path criteria.
\end{abstract}

Keywords - Formation Control, Autonomous Agents, Leader-Follower, Motion Control, Directed Graph

\section{INTRODUCTION}

There are several examples of coordinated team work of mobile agents in nature like food foraging by a group of ants and flocking birds, fish schooling for protection from enemies, etc. These examples give us a lesson that for and automation significantly to contribute in several similar applications in industry. In some particular applications

efficiently by a group of agents if they perform in a collaborative manner. During last thirty years or more this fact has motivated many researchers in the field of control particular task collective effort is more efficient than individual effort. These facts have motivated many researchers in the field of control and automation significantly to contribute in several similar applications in industry. In some particular applications during their motion as whole, autonomous agents (e.g. robots, vehicles, etc.) need to maintain a particular geometrical shape for cohesive motion, called formation which satisfies some constraints like desired distance between two agents, desired angle between two lines joining two agents each. Examples of these types of formations are found in collective attack by a group of combat aircraft, search/rescue/surveillance/cooperative transportation by a troop of robots, under water exploration /under water inspection (like pipeline inspection) by a group of Autonomous Under water Vehicles, attitude alignment of clusters of satellites, air traffic management system, automated highway system etc.

In the area of formation control of autonomous agents like robots the motion control strategies may be either a centralized one or it could be a decentralized control. In centralized mode of control $[1,2]$, the command for all agents of the group are assigned by the central command control board or a designated group leader for monitoring and control of all agents to guide them be placed at desired position. The centralized formation control could be a good scheme for a small group of robots, when it is implemented with a single computer and a single sensor to monitor and control the entire group. Control of large number of robots in a formation requires greater computational capacity, large amount communication. Due to these problems decentralized control is recommended when formation involves a large number of agents. In the decentralized mode of control $[4,5,7]$, one agent of the group can be a leader and others are followers (or each agent of the group can be a leader and follower except a designated group leader and the two outmost agents) and as a follower each agent generates its own commands autonomously (i.e. control law for each agent is provided separately such that each agent works autonomously) based on the relative measurement only from its neighbours without need of an

external supervisor and whole purpose of the formation motion is achieved.

Although two types of basic control strategies are discussed in the last paragraph, motion control scheme for formation may be modified depending upon some factors like agent dynamics, inter-agent information exchange structure, control goals in different applications, etc.

Formations are modeled using formation graph whose each vertex represents individual agent kinematics and each edge represents inter-agent constraint (e.g. desired distance) that must be maintained during motion of formation. Specifically graph is used to represent coordinated behaviour among agents. Depending upon the pattern of information exchange in that coordination, two types of graphs are possible i.e. directed and undirected.

In undirected formation graph both of any pair of agents, constrained by an edge have equal responsibility to satisfy the constraint. As an example, distance between any pair of agents is sensed by both the agents i.e. sensing is distributed. Therefore 
structure of undirected graph suffers from various disadvantages. One of those disadvantages is information-basedinstability [3], which happens due to possibility of difference in distances measured by noisy sensors of any pair of agents. Communication requirement among agents is more. Therefore external observer based centralized control strategy is best suited for undirected formation graph. Control law is mainly focused on rigidity property of formation.

Formation graph is rigid [10] if distance between any pair of agents remains constant during any continuous motion of formation. Mathematically, a representation $p$ of an undirected graph $G(V, E)$ with vertex set $V$ and edge set $E$, a function $p: V \rightarrow \mathfrak{R}^{d}$, is rigid if there exists $\varepsilon>0$ such that for all realizations(due to continuous deformations) $p^{\prime}$ of distance set induced by $p$ and satisfying $d\left(p, p^{\prime}\right)<\varepsilon$ there holds $\left\|p^{\prime}(i)-p^{\prime}(j)\right\|=\|p(i)-p(j)\|$ for all $i, j \in V$.We simply say this phenomenon as congruence relationship between $p$ and $p^{\prime}$ A graph is said to be minimally rigid if it is rigid and if there is no rigid graph having the same vertices but fewer edges.

In directed formation graph, only one (called follower) of any pair of agents, constrained by an edge has responsibility to satisfy the constraint. Therefore decentralized control strategy is best suited for directed formation graph.

Suppose, for a directed graph $G$,desired distances $d_{i j}>0$ for $\forall(i, j) \in E$, edge set where $i, j \in V$, vertex set and a realization $p$, then the following definitions are described.The edge $(i, j) \in E \quad$ is active if $\|p(i)-p(j)\|=d_{i j}$ i,e.if the corresponding distance constraint is satisfied.The position of a vertex $i \in V$ is fitting for any desired distance set $\{d\}$ of $G$ if it is not possible to increase the set of active edges leaving $i$ by changing the position of $i$ while maintaining the positions of other vertices unchanged. Specifically, the position of vertex $i$,for a given realization $p$,is fitting if there is no $p^{\prime} \in \mathfrak{R}^{2}$ for which the condition elaborated next is strictly satisfied $(\{, j) \in E:\|p(i)-p(j)\|=d_{i j} \overbrace{}^{\mathcal{T}}\{(i, j) \in$ $\left.E:\left\|p^{\prime}-p(j)\right\|=d_{i j}\right\}$.A realization of a graph is a fitting realization for a certain distance set $\{d\}$ if all the vertices are at fitting positions for $\{d\}$.A realization $p$ of digraph $G$ is constraint consistent if there exists $\varepsilon>0$ such that any realization $p^{\prime}$ fitting for the distance set $\{d\}$ induced by $p$ and satisfying $d\left(p, p^{\prime}\right)<\varepsilon$ is a realization of $\{d\}$. Realization $p$ of the digraph $G$, desired distances $d_{i j}>0$ for all $\{i, j\}$ is persistent if there exists $\varepsilon>0$ such that every realization (due to continuous deformation) $p^{\prime}$ fitting for the distance set induced by $p$ and satisfying $d\left(p, p^{\prime}\right)<\varepsilon$ is congruent to $p$. A realization is persistent [11] if and only if it is rigid and constraint consistent. A graph is generically persistent if and only if it is generically rigid and constraint consistent.A persistent graph is minimally persistent if it is persistent and if no edge can be removed without losing persistence.

However focus in this paper is in development of control strategy for directed graph based formation. There are several papers in which basics of directed formation graph related issues are discussed [10, 11, 12, 13].

Digraph is called acyclic when no cycle is present in its sensing pattern $[3,11]$. Control scheme for cyclic formation is more complicated than acyclic formation. Minimally persistent formation of autonomous agents may be formed in two ways .First one is leader-follower graph architecture constructed from an initial leader-follower seed by Henneberg Sequence with standard vertex additions or edge splitting [6]. Leaderfollower type minimally persistent graph is always acyclic. Another type of construction by sequence of specific operation elaborated in [12] such that every intermediate construction is also persistent. Minimally persistent graph constructed by this method may have cycles. In this paper control strategies for only leader-follower type formation constructed from sequence of vertex addition is described.

Although a number of research works have been directed in the area of cyclic formation graph, but still there remains scope of further work. In the work [16] Anderson et. al. have proposed a distance preservation based control law when cycles contain in the formation graph.

In most recent works [14, 15] or some previous works [3] distributed control is provided with exploiting gradient control law for each autonomous agent in a formation separately. In [7], formation control strategy of leader-follower and three coleader structures is set up based on discrete-time motion equations considering decentralized approach. In [8], Anderson et al. analyzed control of leader-follower structure with cycles, in continuous domain assuming linearized system for small displacement and stability aspects are also discussed. However, we proceed for the control of leader-follower formation in a different way i.e. our approach is based on optimization of some distances.

In the present work, we consider the motion control scheme of the leader-follower structure type persistent formation in continuous domain. It is based on optimization of a set of nonlinear objective functions corresponding to each agent under a set of equality constraints. It may be noted that for each agent both the objective function and the constraints are different. The control scheme proposed considers a decentralized approach. With advent of high speed computational platforms the solution associated with optimization procedure in the control generation is possible. 
This paper is organized as follows. In section 2, Problem formulation for formation control of multiple autonomous agents has been discussed. Development of control law has been included in section 3 followed by simulation, conclusion in sections 4 and 5 respectively

\section{PROBLEM FORMULATION}

For simplicity, a triangular formation of leader-follower structure with three mobile autonomous point agents, in plane is considered. This triangular formation is shown in Figure 1.where R-1, which has no outgoing edge, represents the leader; $\mathrm{R}-2$, called the first follower which has one outgoing edge i.e. it requires to maintain only one desired distance constraint from the leader and R-3, called the ordinary follower which has two outgoing edges i.e. it requires to maintain two distance constraints, one of which is directed towards leader and other one towards first follower.

The different distance constraints among agents assumed are as follows. $d_{1}$ is the desired distance maintained by first follower from leader, $d_{2}$ is the desired distance maintained by ordinary follower from first follower; $d_{3}$ is the desired distance maintained by ordinary

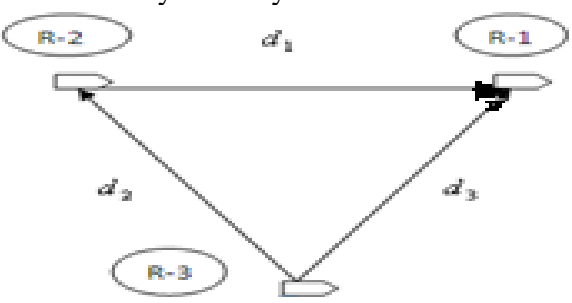

Figure.1 Triangular formation of leader-follower structure. follower from leader.

We assume the desired distances among the agents satisfy triangle inequalities given by

$$
d_{1}<d_{2}+d_{3}, d_{2}<d_{1}+d_{3}, d_{3}<d_{1}+d_{2}
$$

\subsection{Assumption:}

( $i$ ) For simplicity, for each agent ' $i$ ', the kinematic model

[7] of unicycle non-holonomic point agent is considered as

$$
\left(\dot{x}_{i}, \dot{y}_{i}\right)=\left(v_{i} \cos \theta_{i}, v_{i} \sin \theta_{i}\right) \& \dot{\theta}_{i}=\omega_{i}
$$

where $p_{i}(t)=\left(x_{i}(t), y_{i}(t)\right)$ with $i=1,2,3$ denotes, the position of $i^{\text {th }}$ agent and $\theta_{i}(\mathrm{t}) \& v_{i}(t)$ denote the orientation and translational velocity of the $i^{\text {th }}$ agent at each instant of time $(t)$, respectively. $\left(x_{i}(t), y_{i}(t)\right)$ is w.r.t a global coordinate coordinate system.

(ii) each agent can measure its position w.r.t the global coordinate system by proper arrangement of a navigation sensor in each agent (iii) first follower has relative distance and bearing information of leader only and ordinary follower has relative distance and bearing information of leader \& first follower both using an active sensor (e.g. sonar) for each agent (first follower and ordinary follower) w.r.t their respective cartesian coordinate system fixed on the respective point body.

(iv) using their own positions w.r.t the global coordinate system and relative distance and bearing information in (ii) w.r.t respective cartesian coordinate system fixed on their (first follower and ordinary follower) point bodies; positions of other agents can be converted with respect to the global coordinate system

It is intended to provide a control scheme for this triangular formation (starting from a non-collinear arrangement) such that during the motion of three robots for any mission, desired interagent distances are preserved.

\section{DEVELOPMENT OF CONTROL LAW}

The objective of the controller design is intended to develop set of decentralized control laws for overall formation. Hence, for each agent, a separate control law for continuous movement in autonomous manner based on local knowledge only of direction of its neighbours has been considered. The control law for each agent is derived by optimizing the corresponding objective function with given constraints including the desired distance constraints of formation. The unknown variables to be solved, are $\boldsymbol{x}$ and $y$ coordinates (which are continuous function of time) of position (with respect to the global coordinate system) of corresponding agent for which objective function is derived.

\subsection{Assumption:}

(i) Inter-agent distances are sufficiently large so that initial collision among robots can be avoided for almost continuous motion of robots.

(ii) Initially positions of robots are not collinear i.e. condition (1) is satisfied.

(iii) During motion of robots no communication failure occurs among the robots

(iv) There is no time delay and uncertainty in sensing the information

(v) Control input in the form of translational velocity and angular velocity (discussed later in this section) calculated from final and initial position of any agent must be generated by controller of each agent.

Now at the outset, we proceed for development of control law.

When all the agents of given formation completes movement to a new set of position coordinates from an old set of position coordinates during certain period of time(usually very small) such that desired distances among agents are preserved for both set of positions and not any other distance preserving position set is available in between these two position sets during motion, then the movement of formation(as a whole) from the old set to new set of positions is called one complete displacement of formation

Before proceeding to develop the control laws for all agents, it is assumed that at any time $t$ each agent is maintaining its own distance constraints. Then how these agents move to their new positions for a complete displacement is discussed below. 


\subsubsection{Control law for the leader:}

Leader doesn't need to maintain any distance constraint from any other agent in formation.. A specified control action is provided for its dynamics such that it moves along a specified path (trajectory) i.e. each position (at each instant of time $t$ ) coordinate is known (preprogrammed) to computational system of the leader. Suppose, at time $t$ initial position coordinate for leader is assumed as $\left(\left(x_{1 I n}(t), y_{1 I n}(t)\right)\right.$. Let the leader moves to a new position $\left(x_{1 f}(t), y_{1 f}(t)\right)$ i.e. the final position (rest point), in very small period of time $d t$ such that continuity preserves between $\left(x_{1,}(t), y_{1, s}(t)\right) \&\left(x_{1 f}(t), y_{1 f}(t)\right)$ i.e. the distance between these two positions is sufficiently small. This motion of the leader and corresponding movement of the first follower is shown in Figure 2.According to Figure 2 $d \vec{s}_{1}=d x_{1} \hat{i}+d y_{1} \widehat{j}$ where $\vec{s}_{1}(t)$, a vector field along the trajectory curve of the leader. $\hat{i}$ and $\hat{j}$ are unit vectors along $x$ and $y$ direction of the global coordinate system. $d x_{1}=x_{1 f}-x_{1 I n}$ and $d y_{1}=y_{1 f}-y_{1 I n}$.It should be noted that $\left(x_{1 I n}(t), y_{1 I n}(t)\right)$ and $\left(x_{1 f}(t), y_{1 f}(t)\right)$ are always on $\vec{S}_{1}(t)$. Therefore, control input to reach its final position is

$\vec{v}_{1}(t)=\frac{d \vec{s}_{1}(t)}{d t}=\frac{d x_{1}}{d t} \hat{i}+\frac{d y_{1}}{d t} \hat{j}=v_{1 x}(t) \hat{i}+v_{1 y}(t) \hat{j}$

where, $\frac{d x_{1}}{d t}=v_{1 x}(t)$ and $\frac{d y_{1}}{d t}=v_{1 y}(t)$

The translational velocity control input during $d t=\left\|\vec{v}_{1}\right\|=\sqrt{\left(v_{1 x}(t)\right)^{2}+\left(v_{1 y}(t)\right)^{2}}$ meter/sec.

The angular velocity control input ( $\mathrm{rad} . / \mathrm{sec}$ ) during same period of time is

$$
\begin{aligned}
& \omega_{1}(t)=\tan ^{-1}\left|\frac{d y_{1}}{d x_{1}}\right| \text { when is } d x_{1} \text { is }+\mathrm{ve}, d y_{1} \text { is }+\mathrm{ve} \\
& =\left(\pi-\tan ^{-1}\left|\frac{d y_{1}}{d x_{1}}\right|\right) \text { when } d x_{1} \text { is }-\mathrm{ve}, d y_{1} \text { is }+\mathrm{ve} \\
& =-\left(\pi-\tan ^{-1}\left|\frac{d y_{1}}{d x_{1}}\right|\right) \text { when } d x_{1} \text { is }-\mathrm{ve}, d y_{1} \text { is }-\mathrm{ve} \\
& =-\tan ^{-1}\left|\frac{d y_{1}}{d x_{1}}\right| \text { when } d x_{1} \text { is }+\mathrm{ve}, d y_{1} \text { is }-\mathrm{ve} \\
& =-\pi / 2 \text { or } \pi / 2 \text { when } d x_{1}=0 \text { and } d y_{1} \text { is }+\mathrm{ve} \text { or -ve }
\end{aligned}
$$

\subsubsection{Control Law for First Follower:}

It may be noted that first follower has one outgoing edge i.e. it has to maintain only one distance constraint (desired distance $d_{1}$ ) and that is to leader. Initial and final position coordinates for the leader are $\left(x_{1 B t}(t), y_{1 B}(t)\right),\left(x_{1 f}(t), y_{1 f}(t)\right)$ respectively. Then first follower senses the disturbance in position of leader, i.e. it senses error in desired distance constraint $\left(d_{1}\right)$ by sensing the final position of the leader staying at its initial position $\left(x_{2 I n}(t), y_{2 I n}(t)\right)$. It tries to satisfy this distance constraint to leader. Therefore, suppose, it moves to a rest point (final position) $\left\{x_{2 f}(t), y_{2 f}(t)\right)$ at the next instant of time $d t$ after the instant during which the leader moves to its final position. During this movement of first follower, leader is assumed to be stationary at the position $\left(x_{1 f}(t), y_{1 f}(t)\right)$. A condition is given to first follower such that only due to disturbance in position of leader; first follower changes its position .To maintain the cohesive motion with the leader distance of the first follower from the leader must be $d_{1}$ at final position of both the agents. It can be formulated as follows:

$$
\left(x_{1 f}(t)-x_{2 f}(t)\right)^{2}+\left(y_{1 f}(t)-y_{2 f}(t)\right)^{2}-d_{1}^{2}=0
$$

where the values of $x_{1 f}, y_{1 f}, d_{1}$ are known to computational system of first follower. But it is clear from equation (4) that locus of the position of first follower is a circle. Hence, its rest position may be at anywhere on this circle. First follower may take its rest position for which it crosses over the leader and may collide with leader. Undesirable consequence of this phenomenon is ordinary follower may collide with leader and first follower both for maintaining the distant constraints from both of them. Hence to provide a control avoiding this unsafe situation, a restriction to the motion of first follower must be imposed, such that it reaches to a rest point on the circle so that distance between the new and initial position of it is minimum. 


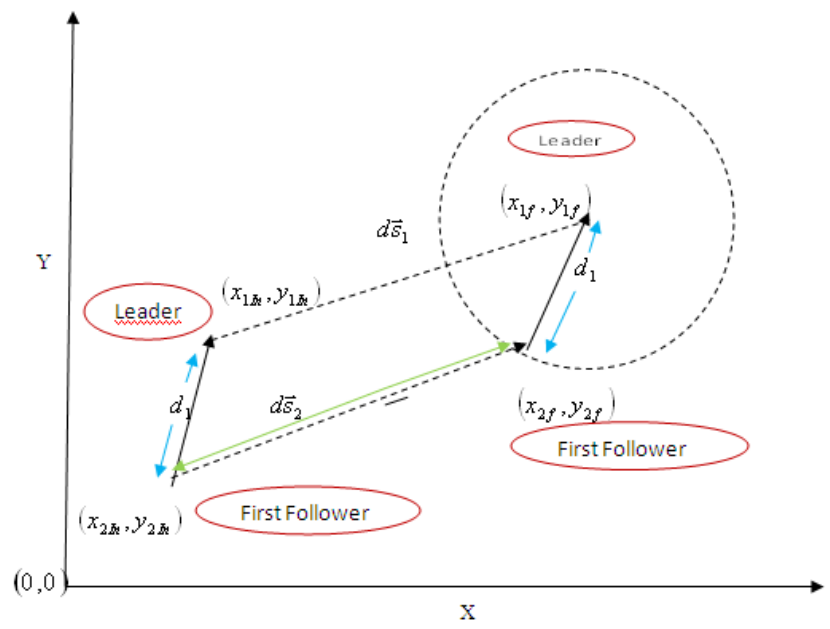

Figure 2. Motion of Leader and First Follower for a very small duration of time $(d t)$

In Figure 2, $\left\|d \vec{s}_{2}\right\|$ is defined as the distance between the final and initial position of first follower. Here $\vec{s}_{2}(t)$, a vector field along the trajectory curve of first follower. It should be noted that $\left(x_{2 I n}(t), y_{2 I n}(t)\right)$ and $\left(x_{2 f}(t), y_{2 f}(t)\right)$ are always on $\vec{s}_{2}(t)$. Then, we have,

$$
\left\|d \vec{s}_{2}\right\|^{2}=\left(x_{2 f}(t)-x_{2 B_{2}}(t)\right)^{2}+\left(y_{2 f}(t)-y_{2 B_{2}}(t)\right)^{2}
$$

Therefore, $\left\|d \vec{s}_{2}\right\|$ must be minimum such that first follower moves along the shortest path to its final position. Hence, the first follower follows the leader maintaining safe motion. Now we intend to propose a control law for motion of first follower satisfying aforesaid conditions. Actually the whole problem may be treated as an optimization problem where minimization of objective function (5) under equality constraint (4) should be performed. And a control law based on this optimization is presented as

$\vec{v}_{2}(t)=\frac{d \vec{s}_{2}}{d t}=\frac{d x_{2}}{d t} \hat{i}+\frac{d y_{2}}{d t} \hat{j}=v_{2 x}(t) \hat{i}+v_{2 y}(t) \hat{j}$

where, $\frac{d x_{2}}{d t}=v_{2 x}(t)$ and $\frac{d y_{2}}{d t}=v_{2 y}(t)$ and $\hat{i}$ and $\hat{j}$ are unit vectors along $x$ and $y$ direction of the global coordinate system.

The

translational velocity

control

input $=\left\|\vec{v}_{2}\right\|=\sqrt{\left(v_{2 x}(t)\right)^{2}+\left(v_{2 y}(t)\right)^{2}}$ meter/s.

The angular velocity ( $\mathrm{rad} / \mathrm{s})$ control input is

$$
\begin{aligned}
& \omega_{2}(t)=\tan ^{-1}\left|\frac{d y_{2}}{d x_{2}}\right| \text { when is } d x_{2} \text { is }+\mathrm{ve}, d y_{2} \text { is }+\mathrm{ve} \\
& =\left(\pi-\tan ^{-1}\left|\frac{d y_{2}}{d x_{2}}\right|\right) \text { when } d x_{2} \text { is }-\mathrm{ve}, d y_{2} \text { is }+\mathrm{ve} \\
& =-\left(\pi-\tan ^{-1}\left|\frac{d y_{2}}{d x_{2}}\right|\right) \text { when } d x_{2} \text { is }-\mathrm{ve}, d y_{2} \text { is }-\mathrm{ve} \\
& =-\tan ^{-1}\left|\frac{d y_{2}}{d x_{2}}\right| \text { when } d x_{2} \text { is }+\mathrm{ve}, d y_{2} \text { is }-\mathrm{ve} \\
& =-\pi / 2 \text { or } \pi / 2 \text { when } d x_{2}=0 \text { and } d y_{2} \text { is +ve or -ve }
\end{aligned}
$$

3.2.3. Control Law for Ordinary Follower: Ordinary follower tries to satisfy two distance constraints i.e. it has two outgoing edges, first one $\left(d_{2}\right)$ is directed towards first follower and second one $\left(d_{3}\right)$ is directed towards leader. Let the leader and first follower have been placed at their corresponding final positions. The ordinary follower senses the disturbances in position of leader and first follower i.e. it senses error in desired distance constraints $d_{2}$ and $d_{3}$ by sensing the final position of the first follower and leader respectively. It tries to satisfy these distance constraints to first follower and leader. Therefore, suppose, the ordinary follower moves to its final position (rest point) at the next time instant of the time ( $d t)$ after the instant during which first follower moves to its final position. During this movement of ordinary follower, leader and first follower are assumed to be stationary at the position $\left(x_{1 f}(t), y_{1 f}(t)\right)$ and $\left(x_{2 f}(t), y_{2 f}(t)\right)$ respectively. At the final position ordinary follower satisfies its distance constraints .Here a condition is given to the ordinary follower such that only when disturbances in positions of both leader as well as first follower (not merely leader) occur; ordinary follower changes its position to final one. This final position is assumed $\left(x_{3 f}(t), y_{3 f}(t)\right)$.Assumed initial position of the ordinary follower is $\left(x_{3 B_{t}}(t), y_{3 B_{4}}(t)\right)$ Now according to the desire distance constraints for it two conditions are to be satisfied, as given below:

$\left(x_{1 f}(t)-x_{3 f}(t)\right)^{2}+\left(y_{1 f}(t)-y_{3 f}(t)^{2}-d_{3}^{2}=0\right.$

$\left(x_{2 f}(t)-x_{3 f}(t)\right)^{2}+\left(y_{2 f}(t)-y_{3 f}(t)\right)^{2}-d_{2}^{2}=0$ 
where, $x_{1 f}, y_{1 f}, x_{2 f}, y_{2 f}, d_{2}, d_{3}$ are known to the computational system of ordinary follower. Hence, $\left\|d \vec{s}_{3}\right\|$ is defined as distance between the final and initial position of ordinary follower. Here $\vec{s}_{3}(t)$, a vector field along the trajectory curve of ordinary follower. It should be noted that $\left(x_{3 I n}(t), y_{3 I n}(t)\right)$ and $\left(x_{3 f}(t), y_{3 f}(t)\right)$ are always on $\vec{s}_{3}(t)$. Then we define,

$\left\|d \vec{s}_{3}\right\|^{2}=\left(x_{3 f}(t)-x_{3 s}(t)\right)^{2}+\left(y_{3 f}(t)-y_{3 A}(t)\right)^{2}$

Actually equations (7) and (8) are equations of two circles. They meet at two different points.

The ordinary follower will follow the leader and first follower maintaining safe motion and moves to any one meeting point such that $\left\|d \vec{s}_{3}\right\|$ is minimum. By maintaining $\left\|d \vec{s}_{3}\right\|$ minimum, ordinary follower moves along shortest path to its final position. Now it is the need to propose a control law for motion of first follower satisfying aforesaid conditions. Actually the whole problem may be treated as an optimization problem where minimization of objective function (9) under equality constraint (7) \& (8) should be performed. And a control law based on this optimization is presented as:

$$
\vec{v}_{3}(t)=\frac{d \vec{s}_{3}}{d t}=\frac{d x_{3}}{d t} \hat{i}+\frac{d y_{3}}{d t} \hat{j}=v_{3 x}(t) \hat{i}+v_{3 y}(t) \hat{j}
$$

Where, $\frac{d x_{3}}{d t}=v_{3 x}(t)$ and $\frac{d y_{3}}{d t}=v_{3 y}(t) \quad$ and

$\hat{i}$ and $\hat{j}$ are unit vectors along $x$ and $y$ direction of the global coordinate system.

The translational velocity control input

$\left\|\vec{v}_{3}\right\|=\sqrt{\left(v_{3 x}(t)\right)^{2}+\left(v_{3 y}(t)\right)^{2}} \mathrm{~m} / \mathrm{s}$

Angular velocity (rad. /s) control input is

$$
\begin{aligned}
& \omega_{3}(t)=\tan ^{-1}\left|\frac{d y_{3}}{d x_{3}}\right| \text { when is } d x_{3} \text { is }+\mathrm{ve}, d y_{3} \text { is }+\mathrm{ve} \\
= & \left(\pi-\tan ^{-1}\left|\frac{d y_{3}}{d x_{3}}\right|\right) \text { when } d x_{3} \text { is }-\mathrm{ve}, d y_{3} \text { is }+\mathrm{ve} \\
= & -\left(\pi-\tan ^{-1}\left|\frac{d y_{3}}{d x_{3}}\right|\right) \text { when } d x_{3} \text { is }-\mathrm{ve}, d y_{3} \text { is }-\mathrm{ve}
\end{aligned}
$$

$=-\tan ^{-1}\left|\frac{d y_{3}}{d x_{3}}\right|$ when is $d x_{3}+\mathrm{ve}, d y_{3}$ is $-\mathrm{ve}$

$=-\pi / 2$ or $\pi / 2$ when $d x_{3}=0$ and $d y_{3}$ is +ve or $-\mathrm{ve}$

Remarks:Hence, from the previous discussion it is concluded that for each complete displacement of considered triangular formation, at the end of first instant of time $d t$ the leader moves to its desired final position. Then at the end of next instant $d t$ (which is the second one) the first follower moves to its final desired position to maintain distance constraints to the leader, during which leader is kept stationary. At end of another instant $d t$ (which is third one) the ordinary follower reaches to its final position to maintain distance constraints to both leader and first follower. Therefore the agents are not reaching their corresponding final position exactly at the same time. Consequently during the period from "after the starting of first instant" and "before the end of third instant" desired distances are not preserved among the agents, rather the first follower and ordinary follower try to form up.So it may be concluded that after every $3^{*} d t$ time, the desired formation is obtained. Therefore, each agent start to move to its new position (to be in a new position set) after every $2 * d t$ time. That is there is a discontinuous motion occurs for every agent. Therefore, for formation of $n$ number of agents, after every $n * d t$ time the desired formation is maintained. Each agent start to move to its new position (to be in a new position set) after every $(n-1) *$

$d t$ time. However, if the $d t$ is chosen as very small we may assume that all the agents reach their corresponding final (new) positions during first instant of time $d t$ (almost same time taken by leader to reach its desired final position) during each complete displacement of formation and continuous motion of formation is maintained .Consequently we may also assume all the agents moves with continuous motion. Simulation results in the next section are also done based on this assumption.

\section{SIMULATION AND RESULTS}

The control laws (3), (6), (10) have been verified successfully via two cases of simulations for specified

formation with consideration of $d_{1}=d_{2}=d_{3}=2 \mathrm{~m}$.

Control laws given in equations (3), (6), (10) require the final position of the corresponding agent at each instant of time during their motion. For the leader, the final position at each instant of time is available as the path is specified for it, but for other agents, these positions must be calculated. To find out the final position at each instant of time $t$, the controller in each case requires optimization (for our algorithm) of a quadratic objective function under one or two quadratic equality constraints as described in section 3. Several optimization methods are available for this purpose. Our choice here is to exploit Sequential Quadratic Programming (SQP) as it is one of the most popular and robust algorithms for nonlinear continuous optimization. The method is based on solving a series of subproblems designed to minimize a quadratic model of the objective subject to a linearization of the constraints. In the proposed controller, the objective functions are chosen as 
quadratic whilst the constraints are taken as non-linear quadratic which can be linearized during course of optimization procedure. At the beginning of each instant of time $t$ (i.e.at the beginning of a complete displacement of the whole formation), the position of each agent is used as the initial position in control law of that particular agent. This position coordinate is also assumed as starting point of that agent's complete iterative procedure (in optimization process using SQP) for finding out its final position. That iterative procedure follows the steps elaborated below.

(i) making a Quadratic Programming (QP) sub problem (based on a quadratic approximation of the Lagrangian function.) using nonlinear objective function and equality constraints

(ii) solving that Quadratic Programming (QP) sub problem at each iteration

(iii) during (ii) updating an estimate of the Hessian of the Lagrangian at each iteration using the BFGS (BroydenFletcher-Goldfarb-Shanno) formula [20, 21]

(iv) Quadratic Programming solution at each iteration performing appropriate Line Search using Merit Function [19, $20,21]$

\subsection{Specific assumptions in different cases:}

\subsubsection{Case A \& B:}

i) In case $A$, initial position coordinates are assumed as (1.5, $1.732),(0.5,0),(2.5,0)$ respectively for leader, first follower and ordinary follower

ii) In the case B initial position coordinates are assumed as $(0,2),(0,0),(1.732,1)$ respectively for leader, first follower and ordinary follower.

iii) Length of each time instant is considered as 0.001 second for simulation during optimization

ii) Distance travelled by leader is assumed to be $1.5 \mathrm{~m}$ in each case

4.1.2. Case $C$ : $i$ ) Initial positions of leader, first follower and ordinary are $(0,4),(0,2),(1.732,3)$ respectively

ii) Leader is assumed to move along a sinusoidal path

The equations that describe that sinusoidal path of the leader is and $\quad x_{1}(t)=0.03 t \quad\left(1 y_{1}(t)=\sin (0.03 t)\right.$

iii) Time travelled by leader is $200 \mathrm{sec}$.

\subsection{Description of results:}

4.2.1.Case A: A linear trajectory motion with constant velocity of $1 \mathrm{~m} / \mathrm{s}$ is provided to the leader. The paths followed by all other agents along with leader during motion of formation are shown in Figure.3. Distances are observed maintained at specified values after every small instant of time $(0.001 \mathrm{sec})$ during motion. Plots of $d_{1}$ vs. $t, d_{2}$ vs. $t$ and $d_{3}$ vs. $t$ are shown in Figure 4, 5 and 6 respectively for motion of formation during first 1.6 second travelling of formation.

4.2.2. Case B: A circular trajectory motion is given to the leader by providing angular velocity at $0.00157 \mathrm{rad} / \mathrm{s}$ and constant translational velocity input at $1 \mathrm{~m} / \mathrm{s}$ to it. Paths of all agents during motion are shown in Figure.7.From the simulation studies of case B, it is found that the distances among agents are maintained at the desired values after every small instant of time $(0.001 \mathrm{sec})$ during the motion of the formation as they were in case A.

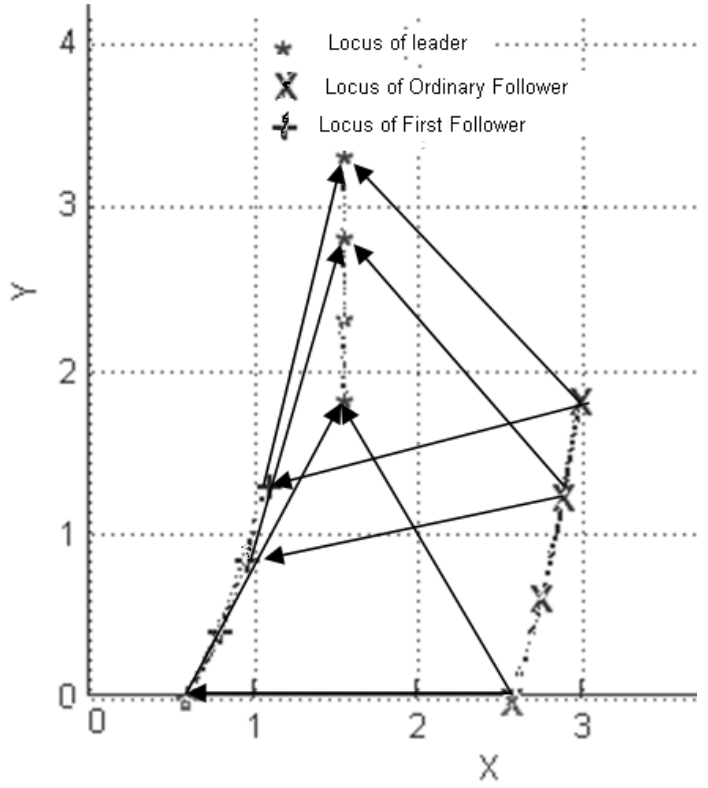

Figure 3. Motion of triangular formation with a linear trajectory motion and constant velocity provided to the leader

\subsubsection{Case C:}

The changes in translational velocity and angular velocity (which are control inputs for leader) along sinusoidal path are shown in Figure 8 and 9 for $150 \mathrm{sec}$.

Paths of all agents during motion of formation are shown in Figure.10.The distances among agents are maintained at the desired values at each instant as they were in case A and B.

Due to space limitations $d_{1}$ vs $t, d_{2}$ vs $t, d_{3}$ vs $t$ are are not shown for case B and C.

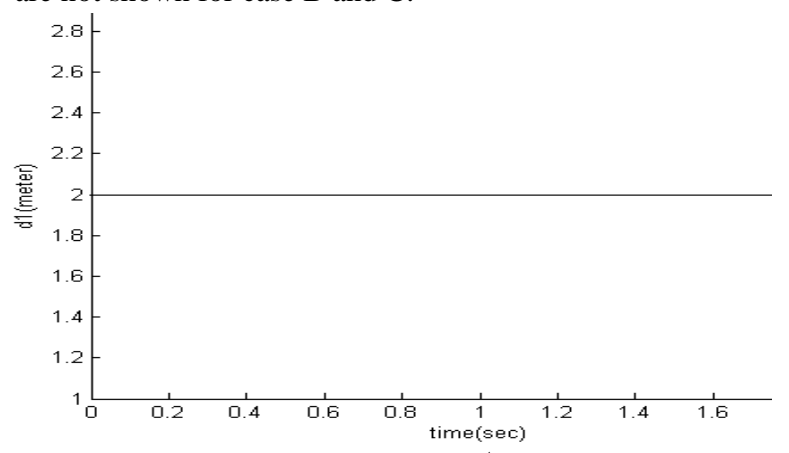

Fig.4.Distance between leader $\& 1^{\text {st }}$ follower vs. time 


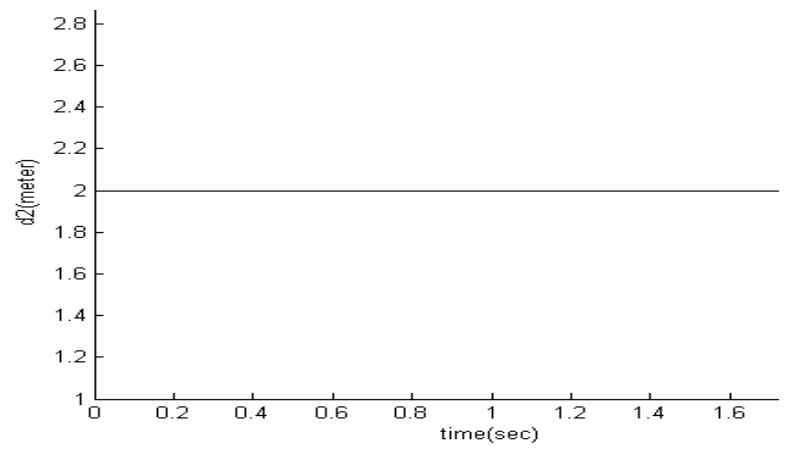

Figure 5. Distance between first follower \& ordinary follower vs. time

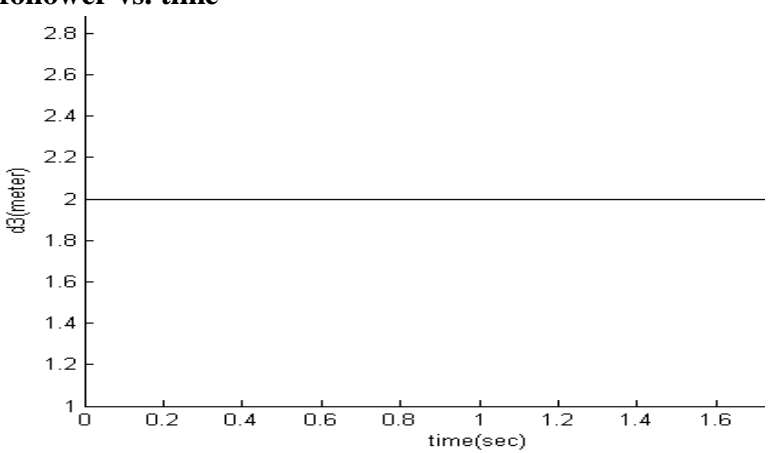

Figure 6. Distance between leader and ordinary follower vs. time

\section{CONCLUSION}

A new algorithm using a set of decentralized control laws based on optimization (using Sequential Quadratic Programming) of distance constraints has been proposed for the motion control of a leader-follower structure type

formation of multiple mobile autonomous agents. Theeffectiveness of the proposed control schemes have been demonstrated through a set of simulation results. During the motion of formation, the inter-agent distances aremaintained at desired values after every specified duration of time and interagent collisions are absolutely avoided. The above described control design for formation of threeautonomous agents may be extended to a large number of agents in the form of leaderfollower structure in which each agent observes the distances of only two or fewer number of neighboring agents to which it needs to maintain distance constraints. The future work includes the the development of a control strategy considering kinematics (other than unicycle non-holonomic point model) of each agent. This control strategy is almost suitable for time

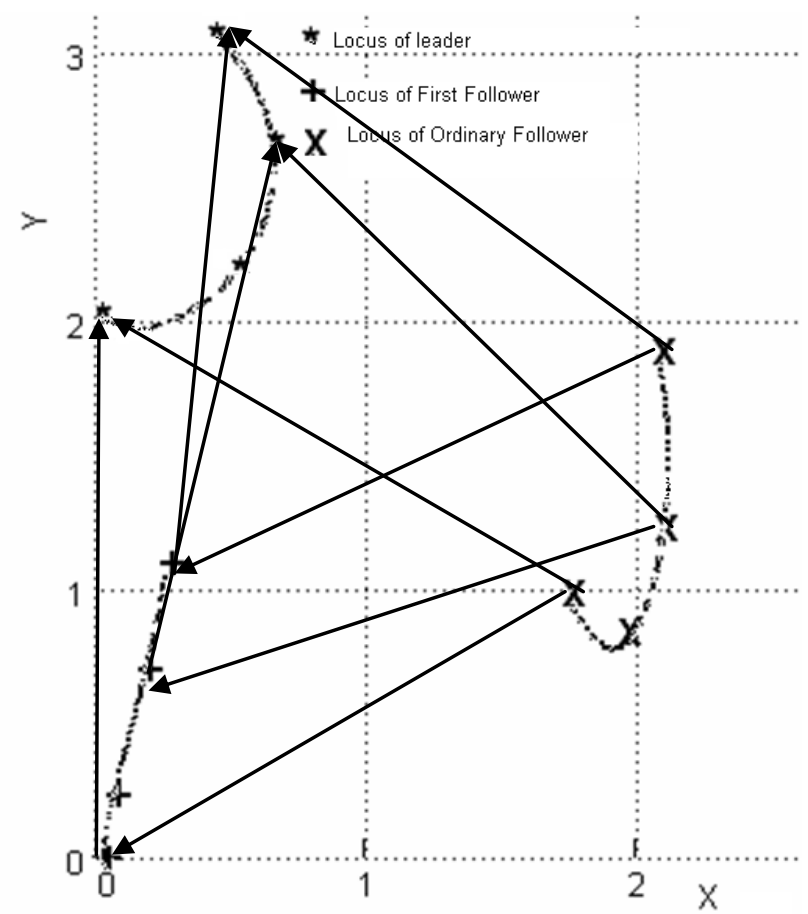

Figure 7. Motion of triangular formation with circular trajectory motion provided to the leader

-critical mission where all vehicles must arrive their final positions exactly at the same time. The immediate task is to develop a control scheme for purely time-critical mission of this type, using our algorithm with some improvement. Stability analysis may be done for our algorithm.

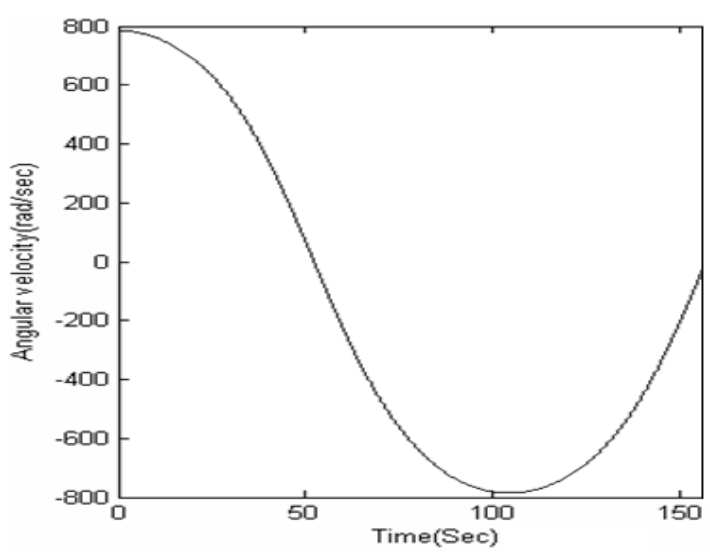

Figure 8. Plot of angular velocity vs time for sinusoidal motion of leader 


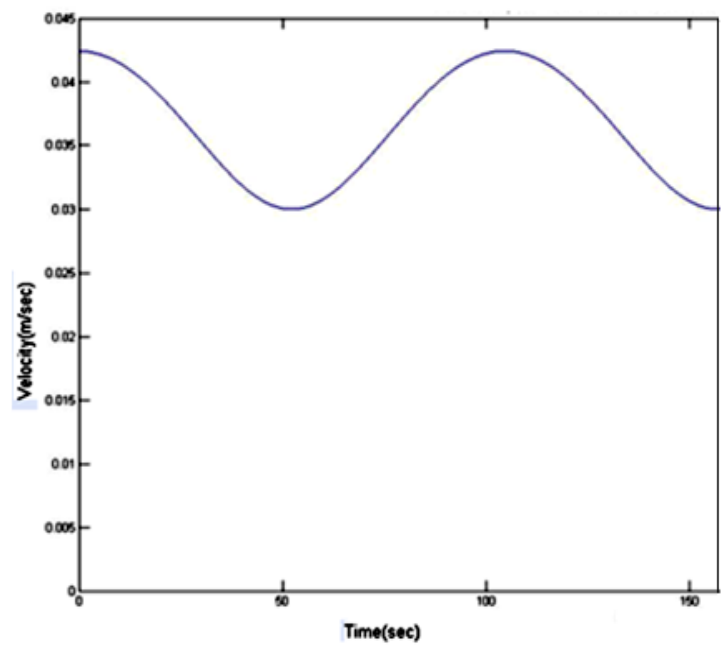

Figure.9. Plot of translational velocity vs. time for sinusoidal motion of leader

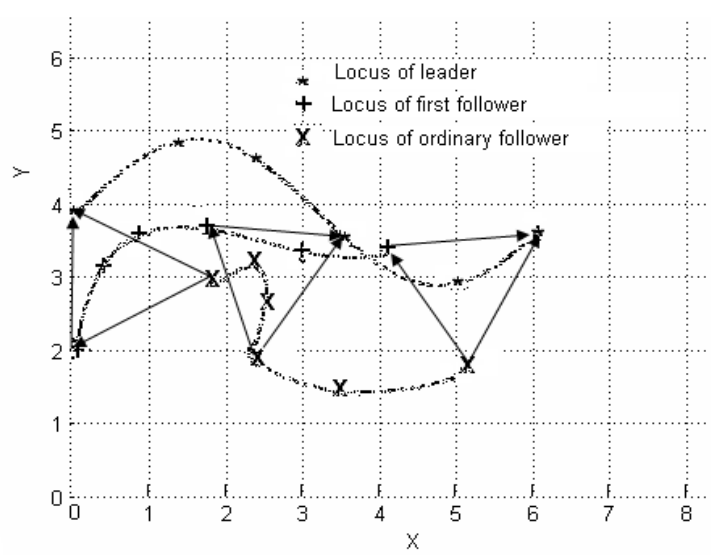

Figure.10. Motion of triangular formation with sinusoidal trajectory motion provided to the leader

\section{REFERENCES}

[1] R. Carelli, C. De La Cruz. F.Roberti, "Centralized Formation Control of Non-Holonomic Mobile Robots", Latin American Applied Research, Vol.36, June 2006

[2]C. De la Cruz, R. Carelli, "Dynamic Modeling and Centralized Formation Control of Mobile Robots", Proc. of 32 nd. IEEE Industrial Electronics Conference, 2006

[3]J. Baillieul and A. Suri, "Information Pattern and Hedging Brockett's Theorem in Controlling Vehicle Formations", in Proc of the $42^{\text {nd }}$ IEEE Conf. on Decision and Control, Vol.1, pp.556-563, December 2003

[4]R. Olfati-Saber and R.M. Murray, "Consensus problems in networks of agents with switching topology and time delays" IEEE Transactions on Automatic Control, Vol.49, No.9, pp.520-1533, September 2004

[5]Jonathan R. T. Lawton, Randal W. Beard, Brett J. Young,"A Decentralized Approach to Formation
Maneuvers", IEEE Transactions on Robotics and Automation, Vol.19, No.6, December 2003 [6]Tolga Eden, Walter Whitely B. D. O. Andersoon, A. Stephen Morse, Peter N. Belhumeur, "Information Structures to Secure Control of Rigid Formations with LeaderFollower Architecture", In Proc. of the American Control Conference, pp. 2966-2971, Portland, Oregon, June 2005.

[7]S. Sandeep, B. Fidan, C.Yu,'Decentralized Cohesive Motion Control of Multi-Agent Formations' in Proc. $14^{\text {th }}$ Mediterranean Conference on Control and Automation, pp. FM1.3 (6 pages), June 2006

[8]B. D. O. Anderson, S. Dasgupta C. Yu," Control of Directed Formations with a Leader-First Follower structure" Proc. of the 46th IEEE Conference on Decision and Control New Orleans, LA, USA, Dec. 12-14, 2007

[9]Wei Ren,"Consensus Based Formation Control Strategies for Multi-vehicle Systems", Proc. of the American Control Conference Minneapolis, Minnesota, USA, June 14-16, 2006

[10]C. Yu, J.M. Hendrickx, B. Fidan, B.D.O. Anderson, and V.D. Blondel, "Three and Higher Dimensional Autonomous Formations: Rigidity, persistence and Structural Persistence," Automatica, vol. 43, pp. 387-402, 2007.

[11]J.M. Hendricks, B.D.O. Anderson, J.-C. Delvenne, and V.D. Blondel, "Directed Graphs for the Analysis of Rigidity and Persistence in Autonomous Agent systems," Int. J. Robust Nonlinear Contr., vol. 17, pp.960- 981, 2007.

[12]J.M. Hendrickx, B. Fidan, C. Yu, B.D.O. Anderson, and V.D. Blondel, "Elementary operations for the Reorganization of minimally persistent formations," in Proc. 17th Int. Sump. Mathematical Theory of Networks and Japan, 2006, pp. 859873

[13]B.D.O Anderson, C. Yu, B. Fidan, J. Hendricks, "Rigid Graph Control Architectures for Autonomous Formations," IEEE Control Systems Magazine, December 2008

[14]M. Cao, A.S. Morse, C. Yu, B.D.O. Anderson, and S. Dasgupta,"Controlling a triangular formation of mobile autonomous agents", In Proc. of the 46th IEEE CDC, pages 3603-3608, 2007.

[15]Laura Krick, Mireille E. Brocke, Bruce A. Fransis, "Stabilization of Infinitesimally Rigid Formations of MultiRobot Networks", International Journal of Control, Volume 82, Number 3, March 2009, pp. 423-439(17)

[16]B.D.O. Anderson, C. Yu, S. Dasgupta, and A.S. Morse," Control of a three co-leaders formation in the plane", Systems and Control Letters, 56:573-578, 2007

[17]Z.Lin, B.Francis, and M. Maggiore,"Necessary and sufficient graphical condition for formation control of unicycles," IEEE Transactions on Automatic Control, Vol.50, No.1, pp121-127, January 2005

[18]Gill, P.E., W. Murray, and M.H. Wright, Practical Optimization, London, Academic Press, 1981. 
[19]Han, S.P., "A Globally Convergent Method for Nonlinear Programming," Vol. 22, Journal of Optimization Theory and Applications, p. 297, 1977.

[20]Powell, M.J.D., "A Fast Algorithm for Nonlinearly Constrained Optimization Calculations," Numerical Analysis, ed. G.A. Watson, Lecture Notes in Mathematics, Springer Verlag, Vol. 630, 1978.

[21]Powell, M.J.D., "The Convergence of Variable Metric Methods for Nonlinearly Constrained Optimization Calculations,"Nonlinear Programming 3 (O.L.Mangasarian, R.R. Meyer, and S.M. Robinson, eds.), AcademicPress, 1978 